\title{
Association between the use of protease inhibitors in highly active antiretroviral therapy and incidence of diabetes mellitus and/or metabolic syndrome in HIV- infected patients: A systematic review and meta- analysis(Article)
}

- Echecopar-Sabogal, J. ${ }^{a}$,

- D’Angelo-Piaggio, L. ${ }^{a}$ Email Author,

- Chanamé-Baca, D.M. ${ }^{a}$,

- Ugarte-Gil, C. ${ }^{\mathrm{a}, \mathrm{b}}$

- View Correspondence (jump link)

- $\quad{ }^{a}$ Escuela de Medicina, Universidad Peruana de Ciencias Aplicadas, Lima, Peru

- ${ }^{b}$ Faculty of Infectious and Tropical Diseases, London School of Hygiene and Tropical Medicine, London, United Kingdom

\begin{abstract}
View references (58)
This systematic review and meta-analysis tries to determine whether there is an association between the use of protease inhibitors (PIs) and the incidence of diabetes mellitus (DM) and/or metabolic syndrome (MS) in HIV-infected patients. A systematic literature search was performed using MEDLINE/PubMed, CENTRAL, LILACS, and EMBASE. Included articles were observational studies published on or prior to November 2015 that met specific inclusion criteria. Pooled relative risks (RRs) and hazard ratios (HRs) were calculated. Nine articles met the inclusion criteria, describing 13,742 HIV patients. Use of PIs was associated with the development of MS (RR: 2.11; 95\% CI 1.28-3.48; p-value 0.003). No association between the use of PIs and development of DM was found: the HR for the incidence of DM among patients using PIs was 1.23 (95\% CI 0.66-2.30; p-value: 0.51) and the RR was 1.25 (95\% CI 0.991.58; p-value 0.06). Use of PIs in HIV-infected patients is associated with an increased risk of MS. No evidence of an increased risk of DM was found. However, because MS is a precursor to DM, it is possible that studies with a longer follow-up duration are needed in order to detect an association between PI use and onset of DM. (C) 2017, (C) The Author(s) 2017.
\end{abstract}

\section{SciVal Topic Prominence}

Topic: $\underline{\text { HIV }}$ | Lipodystrophy | therapy HAART

Prominence percentile: 89.090

\section{Reaxys Database Information}




\title{
Author keywords
}

antiretroviral therapydiabetes mellitusHIVincidencemetabolic syndromeprotease inhibitor

\section{Indexed keywords}

\author{
EMTREE \\ drug terms: Human immunodeficiency virus proteinase inhibitor
}

EMTREE

Articlediabetes mellitusdisease associationdrug usefollow uphighly active medical antiretroviral therapyhumanHuman immunodeficiency virus infected

terms: patientHuman immunodeficiency virus infectionincidencemeta analysismetabolic syndrome Xpriority journalrisk factorsystematic review

- ISSN: 09564624

- CODEN: INSAE

- Source Type: Journal

- Original language: English

- DOI: $10.1177 / 0956462417732226$

- PubMed ID: 28956700

- Document Type: Article

- Publisher: SAGE Publications Ltd 